\title{
A Brief Analysis of Features and Applications of Mobile Phone
}

\section{Animation}

\author{
JunMei Zeng ${ }^{1, a^{*}, \text { QiHui Chang }}{ }^{2, b}$ \\ ${ }^{1}$ Xi'an Polytechnic University, Xi'an,China \\ ${ }^{2}$ Xi'an Polytechnic University, Xi'an,China \\ a445263856@qq.com, bm18609316780@163.com
}

\section{Key words: Mobile phone animation Popularity Impromptu characteristics Interactivity}

\begin{abstract}
With the development of wireless Internet technology, mobile media, which is considered as the fifth media, has its great advantages on mobility and interactivity. Not only are the creation of mobile phone animation and its unique advantages popular with a number of animation fans but also they attract more people's attention. Combining animation with mobile media, this paper will analyze and summarize features and application classification of mobile phone animation to learn and explore the definition and features of mobile phone animation from multi-perspectives.

The birth and development of digital and Internet technology in recent years provide a number of opportunities and platforms for not only the animation creations but also the animation industry. Before the appearance of new media, such as Internet, digital television, and mobile phone, animation art contains two main means of communications media --traditional films and televisions. It is reasonable to claim that film media has several limitations as it restricts the audiences and requires the audiences to see the same film in the same place during a specific time. Compared with film media, television media is less constrained since it provides the audiences with a number of television channels, indicating that this media could be more selective; however, the time of broadcast is scheduled.

Being considered as a tool of entertainment and communication, not only are smartphones used to strengthen interaction and contact but also their function of entertainment becomes the first factor that consumers would take into account. Mobile phone media nowadays is on the way across media and industry, such as communication, Internet, entertainment, and traditional media, combing mobile phone media with animation art. This not only expands the business of mobile phones and their functions but also explores opportunities for the development of animation industry.
\end{abstract}

\section{The definition of mobile phone animation}

As one of the functions of mobile phones, mobile phone animation does not simply mean the combination of mobile phones with animation. The so-called mobile phone animation refers to the animations that are made according to some standards; in other words, most of the works are video files which code as AVI, WMA, MPEG4, and MPEG2 with the resolution of $720 \times 576$, the frame rate of 25 , and the code rate of $4 \mathrm{Mbps}$ for videos and $128 \mathrm{Kbps}$ for audios, etc ${ }^{[1]}$. It uses the Internet to develop animated content that is $3 \mathrm{D}$ interaction with users, provides different forms, such as downloading and forwarding via the mobile Internet, and offers animation product for users through the electronic terminals of mobile phones and various electronic devices. Mobile phone animation does not simply indicate the placement of traditional animation, cartoon, and games in mobile phones; additionally, it refers to a type of mobile business which contains a form of animation ${ }^{[2]}$. Compared with traditional animation, the process of producing mobile animation is easier and the types of playing media are more abundant; moreover, mobile animation is more interactive as users are able to watch, communicate, and interact via their phones wherever and whenever. With the 
improvement of technology and features of mobile phone animation, processing multiple media, such as images, music, and videos on mobile phones becomes easier; in addition, the adoption of vehicle display, iWatch, and iTV contributes greatly to the extension of mobile phone animation.

\section{The features of mobile phone animation}

Mobile phone animation has its own features on the spread of its content, and its methods of spread are also different from traditional ones; therefore, mobile phone animation not only combines the spread of traditional animation but also reflects its unique features, and the connection and development between mobile phone animation and traditional animation are well revealed by these features. Mobile phone animation, which integrates some features of mobile media, has the following features that are different from traditional animation:

1) Popularity

Animation is usually considered as the privilege of children, indicating that only children enjoy watching animations. This is the reason why some animation works tend to be lower age on creation patterns and styles; in other words, animation is produced only for children. However, this is not true. Most mobile phone users are from 18 years old to 35 years old, indicating that the user community is composed of a more mature population, and most of these users would like to choose animation works according to their personal preferences. With the appearance of mobile media, the illusion that animation is only for children has been gradually changed, and the gap between animation and adult customers has been shortened, filling in the blanks of adult animation. This also encourages the development of specific and diversified animation. In addition, some thoughtful and meaningful animation - experimental animation and adult animation, should be promoted in order to rejuvenate and enrich the content of mobile phone animation with creativity and characteristics. This will not only meet the needs of users on enjoying animation but also meet the demand of mobile media on entertainment.

2) Impromptu characteristics

Portability, as one feature of mobile phones, decides that mobile phone animation is able to be optional; in other words, users are able to watch the mobile animation anytime and anywhere, as long as they would like to. This feature, which is quick and immediate, increases the efficiency of acceptance and initiative, indicating that watching mobile animation is no longer limited by the regular time, places, and other conditions. In short, users are able to watch anything they enjoy at anytime, such as on their trails, on the subway, during their spare time, etc.

3) Interactivity

Interactivity is regarded as the most significant feature of spread that differs digital media from traditional media. Compared with traditional media, mobile phone animation is more interactive, and it is more convenient for users to take advantage of its interactivity to adapt to the high rhythm of modern life. Most of this interactivity is embodied in the remaining imagination which is provided for user to create their own derivative works; moreover, users are able to interact with their friends, showing their favorite clips through the push on various social platforms. Nowadays, a number of softwares, which are convenient and easy-to-use for producing short films, are provided in mobile applications. Through these applications, users are able to recreate their favorite pictures, characters, and clips, and then share with their friends. Therefore, having this active choice and operation will not only raise users' interests but also have effects on information dissemination and propagation.

4) The property of freedom

The property of freedom is well reflected in this modern society. Compared with traditional media, users of mobile phone animation will not accept passively, and this mobile phone animation provides its users with a property of freedom that users are allowed to decide time, order, and preference. In other words, users are allowed to choose clips and episodes of an animation work according to their own preferences. This provides users with a wider range of choices and encourages users to explore their favorite animation, feeling free to acquire information. 


\section{The application classification of mobile phone animation}

The so-called mobile phone animation actually refers to the applications and content of animation in mobile phones. The popularity, portability, interactive, and the property of freedom of mobile phone animation differ it from traditional television and mesh animation, indicating that mobile phone animation is more abundant and interactive on its patterns. In the meanwhile, more and more users are attracted to experience mobile phone animation as some features of mobile phone animation, such as having large amounts of information, being entertained, and being targeted, are explored. Based on the continuous development of multimedia and new media, the application classification of mobile phone animation becomes richer from the earliest on-off animation and multimedia message to current mobile applications, such as mobile game, dynamic picture, and interactive animation.

1) On-off animation

The earliest on-off animation, which is as short as a few seconds, actually refers to the animated image of mobile phone companies, and it aims at interpreting the main concepts of mobile phone brands. For example, the brand of Nokia is remembered quickly by the public since the onoff animation is classic. As smartphones fully cover the mobile phone market, the thematic interface that goes with mobile phones can no longer meet users' needs; therefore, in order to meet personal demand, the function of mobile theme, which allows users to download and own different themes, for instance, static or dynamic, and cartoon or painting, emerges rapidly.

2) Multimedia message

Multimedia message is one application that users prefer and commonly use after the emergence of mobile phone animation. Compared with ordinary text message, multimedia message not only allows users to send text message but also provides users with various message formats, for example, the dynamic images. The way to develop multimedia message is quite simple as most multimedia messages are organized by several changing images or figures which are designed in the GIF format and have some add-on sound effects, developing some short films that are similar to animation videos, but this multimedia message can only last for a few seconds. However, as one of the applications, multimedia message becomes increasingly playful through sharing different types, for instance, the weather forecast and newsletters.

3) Apps

Many cartoonists nowadays make use of valuable resources of animation in their hands or add some skillful animation in their own made characters, producing some simple but interesting dynamic images or expression packages. Although not many plots are included, this process do creates a format that encourages people to enjoy and accept it passively. In addition, with the development and improvement of mobile phone applications, the production of mobile phone animation develops a closer relationship with the public as developers adopt many easier operating systems to encourage the public to join in the creation of mobile phone animation. For example, an application named MomanCamera was popular a while ago since it can create a series of dynamic expressions which are cute, funny, and exclusive according to users' selfies and their preferred dynamic templates.

4) Mobile clients

Through client applications, the size of images and the way mobile animation gets displayed match the screen of mobile phones; in addition, the use of animation (zoom in, zoom out, translation, and font magnification) and even some special effects (voice, vibration, and screen dithering) increases the vividness of images and visual enjoyment as well. For example, many Japanese mobiles own some built-in client applications for comic, and this is one important reason that why Japanese comic becomes successful. Therefore, the installment of clients is applicable for different forms of animation, and the excellence of mobile animation is well demonstrated through the profound processing and display according to the animation contents. To animation categories, the client service plays an irreplaceable role in the long-running and mid-sized animation, so a number of mobile animations are in strips. For instance, some Japanese comics are serialized with hundreds of episodes (the so-called "strips"). Being similar to TV series, the serialization plays an 
important role in attracting mobile users. Moreover, the clients are applicable to the short-short animation as they are able to provide better experience of reading, and only based on clients, the long-running animation is able to assemble users and to form a real customer segment, developing a stable and sustaining source for profits. However, from the aspects of product design, user experience, and animation content, etc. there are only a few excellent and developed clients currently, such as Manke, Blink, and Comicsviewer.

5) Interactive games

The majority of users of mobile phone animation are mobile game players, but the previous games such as World of Warcraft is larger competitive game which limits players on computers; however, the appearance of mobile games realizes the idea that users are allowed to play games anytime and anywhere. From the original and simple stand-alone games to interactive mobile games nowadays, interactivity is greatly added to mobile games, for example, the Rhythm Master from the WeChat, the Happy Xiaoxiaole from the Snap Pea, and many other "pass through" games, and through challenging, ranking, and other methods, more players are encouraged to join in these games. Moreover, through raising players' attractions and improving visual effects, the preference and acceptance on mobile phone animation have been largely increased.

\section{Conclusion}

Being as the "fifth media" of new media, mobile phone owns a large number of users. With the appearance and development of mobile animation, the forms of animation art become more abundant, not only providing more conditions for future development, but also offering another model for the development of animation industry. Although there are some problems, such as the immaturity of profit models and the lack of creative talents, mobile animation, as a whole, has a bright prospect and wide space for development. However, for the same mobile phone animation, some problems exist on the traffic limit and rates. Being as one form of entertainment, mobile phone animation shares a high consumption on traffic; therefore, it is necessary to consider how to save traffic and rates for customers, for instance, paying attention to compressed files during production and reminding users to download or cache under the WiFi status.

From the simplest on-off animation to the engaging interactive mobile games, current mobile phone animation has already joined in mobile media from multi-aspects; additionally, animation, which shows strong expressive force and owns abundant information, is well displayed through mobile media. Moreover, the special significance of animation art is well reflected through mobile phone animation. On the other hand, in this era of mobile phone and network media, mobile phone animation not only enjoys more space but also changes people's life through its unique features and leads a new lifestyle as well. Therefore, mobile phone animation is a project which deserves further study and development. In conclusion, this paper has studied and analyzed the features and applications of mobile phone animation from multi-aspects, hoping not only to develop a referential meaning to further creations and researches on mobile phone animation but also contributing to the development of Chinese mobile animation.

\section{Reference:}

[1] Information on.http://baike.baidu.com/view/11671798.htm

[2] Li Sida,introuction ti new media animation[J].Journal of tsinghua university press.2013.7

[3] Zhou Jiansen,zhangyu.Mobile phone animator[J].Science and culture. 2007(03)

[4] He Jianping, new media and the Chinese animation industry interactive mechanism study[J].Journal of contemporary flim.2007(05)

[5] Ji Shizhang,xianglisheng,shenqian,zhangquan.Interaction based on SMIL animation technology[J].Computer and information technology. 2006(14)

[6] Zhang zhengping,zhangbaozhu.Animation industry combined with the new media development[J].Journal of contemporary media.2007(1)

[7] Zhu haisong, The fifth media:focus media and directional communication under wireless marketing[M].Guangdong economic press.2005.1

[8] Kuang wenbo,Ineroduction to the handset media[M].Renmin university of China publishing house.2006.1 
[9] Jin yuanpu,Introduction to animation creative industry[M].Higher education press 2012.2 